\title{
Functional Magnetic Resonance Imaging of Effects of a Nicotinic Agonist in Schizophrenia
}

\author{
Jason R Tregellas*,', Ann Olincy', Lynn Johnson', Jody Tanabe', Shireen Shatti', Laura F Martin', \\ Debra Singel', Yiping P Du', Ferenc Soti', William R Kem² and Robert Freedman' \\ 'Department of Psychiatry, Denver VA Medical Center VISNI 9 MIRECC and University of Colorado Denver, Aurora, CO, USA; ${ }^{2}$ Department of \\ Pharmacology and Therapeutics, University of Florida, Gainesville, FL, USA
}

\begin{abstract}
3-(2,4-Dimethoxybenzylidene)-anabaseine (DMXB-A) is a partial agonist at $\alpha 7$-nicotinic acetylcholine receptors and is now in early clinical development for treatment of deficits in neurocognition and sensory gating in schizophrenia. During its initial phase 2 test, functional magnetic resonance imaging ( $\mathrm{fMRI}$ ) studies were conducted to determine whether the drug had its intended effect on hippocampal inhibitory interneurons. Increased hemodynamic activity in the hippocampus in schizophrenia is found during many tasks, including smooth pursuit eye movements, and may reflect inhibitory dysfunction. Placebo and two doses of drug were administered in a random, double-blind crossover design. After the morning drug/placebo ingestion, subjects underwent fMRI while performing a smooth pursuit eye movement task. Data were analyzed from 16 nonsmoking patients, including 7 women and 9 men. The $150-m g$ dose of DMXB-A, compared with placebo, diminished the activity of the hippocampus during pursuit eye movements, but the 75-mg dose was ineffective. The effect at the 150-mg dose was negatively correlated with plasma drug levels. The findings are consistent with the previously established function of $\alpha 7$-nicotinic receptors on inhibitory interneurons in the hippocampus and with genetic evidence for deficits in these receptors in schizophrenia. Imaging of drug response is useful in planning future clinical tests of this compound and other nicotinic agonists for schizophrenia.
\end{abstract}

Neuropsychopharmacology (2010) 35, 938-942; doi:I0.1038/npp.2009.196; published online 2 December 2009

Keywords: schizophrenia/antipsychotics; imaging; acetylcholine; hippocampus; eye movements; DMXB-A

\section{INTRODUCTION}

A desirable step in the early evaluation of a new drug is to show activity in patients that validates the drug's effects on its putative neurobiological mechanism. This step is not always achievable for psychotropic drugs, because brain mechanisms are generally inaccessible for direct study. Imaging of brain activity is a potentially useful strategy for determining if drug effects in the brain are consistent with the putative mechanism of action. 3-(2,4-Dimethoxybenzylidene)-anabaseine (DMXB-A) is a partial agonist at human $\alpha 7-$ nicotinic acetylcholine receptors (Briggs et al, 1995; De Fiebre et al, 1995; Kem et al, 2004). Its activity in animal models of neurocognitive and sensory gating deficits suggested that it might enhance cognition in schizophrenia (Stevens et al, 1998; Martin et al, 2004). As part of a phase 2 clinical test of that hypothesis, patients who met magnetic

*Correspondence: Dr JR Tregellas, Department of Psychiatry, University of Colorado Denver School of Medicine, 1300 I East 17th Place, Building 500, MS F546, Aurora, CO 80045, USA,

Tel: + I 303724 6232, Fax: + I 303724 6227. E-mail: jason. tregellas@ucdenver.edu

Received 2 September 2009; revised 21 October 2009; accepted 27 October 2009 resonance imaging (MRI) safety requirements participated in functional magnetic resonance imaging (fMRI) studies to attempt to document the neurobiological effect of the drug.

Hippocampal hyperactivity was first observed during SPECT imaging of eye movement in patients with schizophrenia and has since been shown using various paradigms and imaging modalities (Malaspina et al, 1999; Tregellas et al, 2004). The hippocampus responds to environmental stimuli, and its hyperactivity is consonant with the patients' self-report of stimulus flooding (Sobotka et al, 1997). A presumptive mechanism of this hyperactivity is the loss of function of hippocampal inhibitory interneurons, which have a markedly high expression of $\alpha 7$-nicotinic receptors, and are depolarized by $\alpha 7$-nicotinic agonists (Breese et al, 1997; Frazier et al, 1998). We hypothesized that activation of hippocampal interneurons through these receptors would reduce the activity of hippocampal pyramidal neurons. Individual neuronal responses cannot be measured in human brain; therefore, hemodynamic response was chosen as an indirect measure. A previous study of DMXB-A had used electrophysiological evoked responses for this purpose and found that DMXB-A increases neuronal inhibition (Olincy et al, 2006). 


\section{PATIENTS AND METHODS}

\section{Patients}

Patients who met the DSMIV criteria for schizophrenia received 1 month each of placebo, DMXB-A at a 75-mg dose, and a 150-mg dose in randomized, double-blind crossover trial detailed below. Patients were simultaneously treated with their own neuroleptic drug, because its antidopaminergic activity was considered a distinctly different therapeutic principle. There were no effects of neuroleptic drug type or dose on the outcome of the trial. Only nonsmoking patients were studied to prevent possible interference of the effects of DMXB-A by nicotine. Other exclusion and inclusion criteria are detailed in a previous report, which also presents the clinical and neurocognitive outcomes and safety data (Freedman et al, 2008). The trial was approved by the institutional review board and the FDA. Primary neurocognitive and clinical outcomes were specified in the Clinical Trials Registry (NCT00100165). Patients gave their informed consent.

Twenty patients agreed to participate in the fMRI study; of them, two were excluded because of excess movement $(>1.5 \mathrm{~mm})$ during fMRI scans. One subject did not complete all scanning sessions and data from one subject was not useable due to technical problems. Data were analyzed from 16 patients, including 7 women (average age of 50.7 years, SD: 8.54) and 9 men (average age of 42.3 years, SD: 8.96). Nine patients were treated with atypical neuroleptics, two with typicals, and four with clozapine; one subject was unmedicated.

\section{Experimental Drug Protocol}

DMXB-A was synthesized and placed into capsules as previously described (Freedman et al, 2008). Identicalappearing placebo capsules also were prepared. After 1 week of screening, patients received 1 week of placebo to assess compliance. Then they received a baseline assessment of clinical measures, including the BPRS (Overall and Gorham, 1962), the SANS (Andreasen, 1982), and the Simpson-Angus Rating Scale for assessment of extrapyramidal symptoms (Simpson and Angus, 1970). Neurocognition also was evaluated and has been reported previously (Freedman et al, 2008).

The patients were assigned to 4 weeks of twice-daily placebo, $75 \mathrm{mg}$ b.i.d. of DMXB-A, or $150 \mathrm{mg}$ b.i.d. of DMXB-A. Both patients and investigators, except for the pharmacist and biostatistician, were blind to drug identity. All patients received each treatment in a balanced crossover design. The three treatment arms were separated by 1-week washout periods, during which the patients received placebo. Compliance with medication as judged by capsule counts exceeded 90\%. The fMRI session detailed below, along with the clinical and neuropsychological assessments, was repeated at the end of each 4-week treatment arm, immediately after the morning dose of drug. On the morning of the fMRI scan, patients came to the imaging center before their first dose of drug. They were administered the drug by an investigator to allow imaging to occur within 90 min of drug ingestion, near the predicted peak of plasma levels. Physiological measures, including pulse rate and blood pressure, also were obtained for safety and to confirm that the fMRI measure was unrelated to generalized vascular changes.

\section{Plasma Drug Level Assays}

Plasma specimens for drug level assays were obtained 2.25-2.50 h after the first morning dose on the 4 th week of each treatment arm. The specimens were analyzed for DMXB-A by high-performance liquid chromatography as previously described (Mahnir et al, 1998; Kem et al, 2004; Olincy et al, 2006). The 4-hydroxy metabolite of DMXB-A also was detected in the samples, but was generally below the level of reliable quantification.

\section{MRI Methods}

Before functional imaging, a high-resolution, T1-weighted $3 \mathrm{D}$ anatomical scan was acquired for each subject. Functional images were acquired with a gradient-echo T2* blood oxygenation level-dependant (BOLD) contrast technique, with $\mathrm{TR}=2000 \mathrm{~ms}, \mathrm{TE}=30 \mathrm{~ms}, \mathrm{FOV}=220 \mathrm{~mm}^{2}$, $64 \times 64$ matrix, 30 slices, $4 \mathrm{~mm}$ thick, no gap, angled parallel to the planum sphenoidale using a quadrature head coil. In addition, one IR-EPI ( $\mathrm{TI}=505 \mathrm{~ms}$ ) volume was acquired to improve co-registration between the echo planar images and gray matter templates used in pre-processing.

Head motion was minimized using a VacFix headconforming vacuum cushion (Par Scientific A/S, Odense, Denmark). MR-compatible goggles (Resonance Technology, Northridge, CA, USA) were used for visual stimuli.

\section{fMRI Paradigm}

fMRI data were acquired as patients performed a smooth pursuit eye movement task described previously (Tanabe et al, 2002). Briefly, the task consisted of visually tracking a small white dot that moved horizontally back and forth over a visual angle of $26^{\circ}$ at a constant velocity of $16.7^{\circ}$ per second followed by a $700-\mathrm{ms}$ fixation period at the edges. Patients were asked to 'follow the dot, wherever it goes.' The paradigm used a block design with three 30 -s cycles of task alternating with and rest, totaling $3 \mathrm{~min}$, for each of three runs. During 'rest,' patients were asked to 'look straight ahead' at a black screen. Ten additional seconds of task with scanning preceded each run as an equilibration period for the functional images.

\section{fMRI Data Analysis}

Data were analyzed using SPM5 (Wellcome Department of Imaging Neuroscience, London). Data from each subject were realigned to the first volume, normalized to the Montreal Neurological Institute gray-matter template, using a gray-matter-segmented IR-EPI as an intermediate to improve registration, and smoothed with an $8-\mathrm{mm}$ FWHM Gaussian kernel. Data were modeled with an HRFconvolved boxcar function, using the general linear model in SPM5. A 128-s high-pass filter was applied to remove low-frequency fluctuation in the BOLD signal.

To account for both within-group and within-subject variances, a random effects analysis was implemented. 
Parameter estimates for each individual's first-level analysis (SPM contrast images) contrasting 'task' to 'rest' were entered into a second-level analysis using the general linear model. Planned $t$-contrasts were used to test the hypothesis that compared with placebo, task-related response in the hippocampus would be reduced during the DMXB-A condition. The hippocampus was chosen as the region of interest (ROI) because this region previously was shown to be hyperactive during the task in schizophrenia, and showed reduced activity after nicotine administration (Tregellas et al, 2004, 2005). Results were corrected for multiple comparisons with the small volume correction (SVC) based on a $10-\mathrm{mm}$ diameter sphere centered at $x=30, y=-15$, and $z=-14$, the hippocampal location in which effects of nicotine on hippocampal response during eye movements were previously observed in patients with schizophrenia (Tregellas et al, 2005).

\section{RESULTS}

Compared with placebo, $150 \mathrm{mg}$ b.i.d. DMXB-A was associated with less activity in the right hippocampus during the smooth pursuit eye movement task $(t=4.50$, d.f. $=15$, $p<0.004$ ) (Figure 1). BOLD responses for individual patients, in terms of percentage signal change relative to the global mean at the local maximum, are shown in Figure 2. No regions of greater response during $150 \mathrm{mg}$ DMBX-A compared with placebo were observed between groups. In addition, no significant differences were observed between placebo and the 75-mg DMXB-A dose.

Plasma drug levels of $150 \mathrm{mg}$ DMXB-A were inversely correlated with hemodynamic responses in the hippocampus $(t=3.21$, d.f. $=15, p=0.043$ ) (Figure 3 ). No significant correlations were observed between drug-related changes in hippocampal response and clinical measures, including the SANS total score and BPRS total score. Similarly, no correlations were observed between neuronal responses and vascular measures, including pulse rate, systolic and diastolic blood pressure. Detailed descriptions of the therapeutic effects, neurocognitive effects and the safety of DMXB-A have been reported previously (Freedman et al, 2008).

\section{DISCUSSION}

The effects of DMXB-A in the hippocampus are similar to those that we previously observed with acute administration of nicotine in patients with schizophrenia (Tregellas et al, 2005). The location of diminished activity in the hippocampus is similar, as well as the magnitude of the effect. DMXB-A therefore appears to be acting as a nicotinic agonist. Nicotine potently activates and desensitizes $\alpha 4 \beta 2$ type nicotinic receptors at very low brain concentrations; at much higher concentrations, it similarly affects $\alpha 7$-nicotinic receptors. As DMXB-A selectively activates $\alpha 7$-nicotinic receptors in vitro and in vivo (De Fiebre et al, 1995; Stevens et al, 1998), the current results suggest that the previously observed effect of nicotine may be mediated also through this nicotinic receptor subtype. CHRNA7, the gene for the $\alpha 7$-nicotinic receptors subunit, has previously been linked to loss of cerebral inhibition in schizophrenia, which
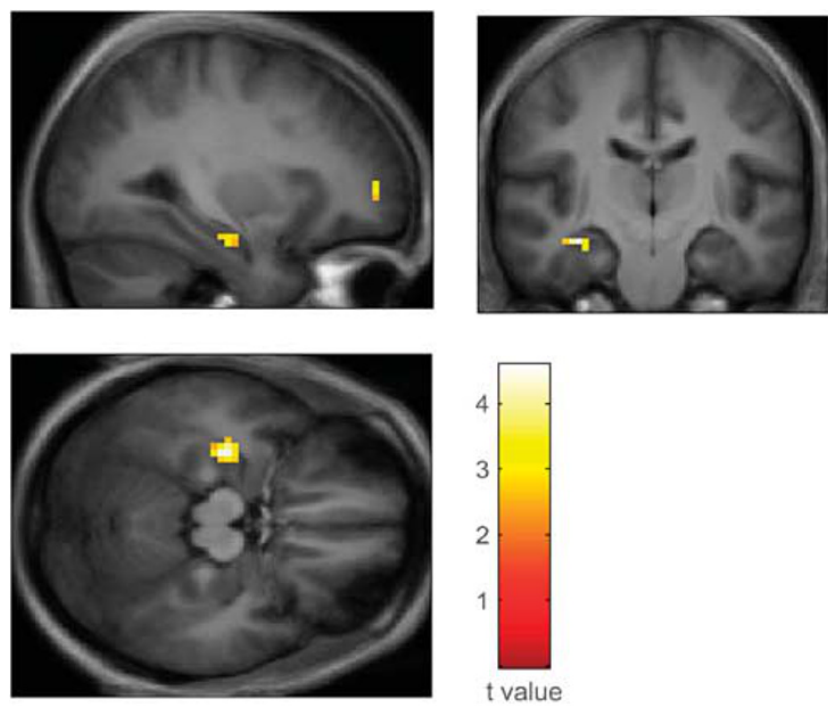

Figure I Less task-related activity in the right hippocampus in patients with schizophrenia treated with $150 \mathrm{mg}$ DMXB-A compared with placebo. Statistical map thresholded at $p<0.01$ for visualization and overlaid onto the group average anatomical image. Data are shown in the radiological convention ( $R$ on $L$ ).

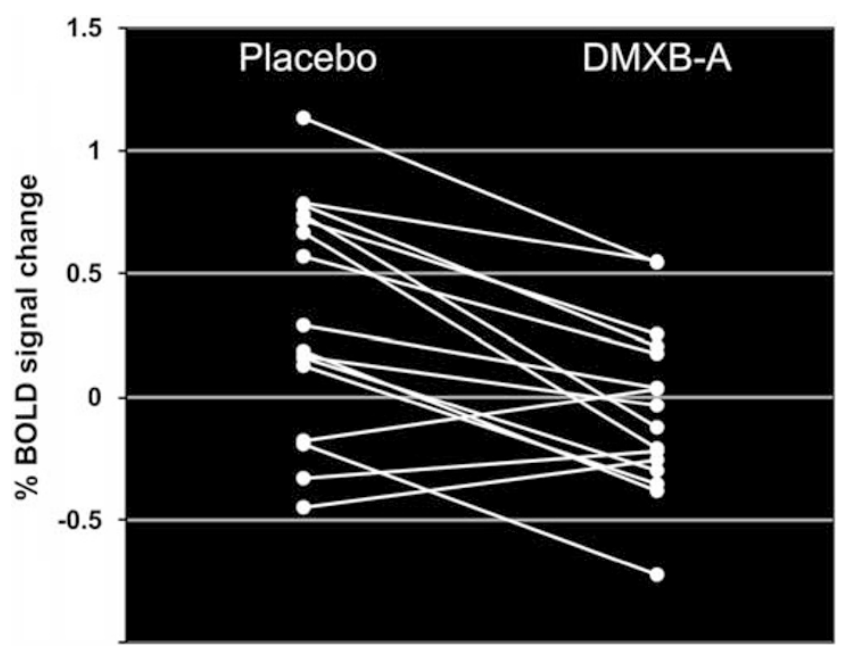

Figure 2 Individual subjects' hippocampal responses during treatment of $150 \mathrm{mg}$ DMXB-A and placebo.

supports the role of $\alpha 7$-nicotinic receptors on cerebral inhibitory interneurons in schizophrenia (Freedman et al, 1997).

A potential pitfall of using fMRI for monitoring drug studies is that the drug may have generalized effects on hemodynamic activity that is misinterpreted as changes in brain activity. In this study, pulse and blood pressure were monitored during DMXB-A administration; no generalized changes in hemodynamic activity were observed.

The utility of this imaging information, in addition to specifying mechanism, is to answer several questions about DMXB-A therapy. First, we have used the same lower and higher doses for phase 1 and phase 2 studies in schizophrenia, because of a possible indication of an 

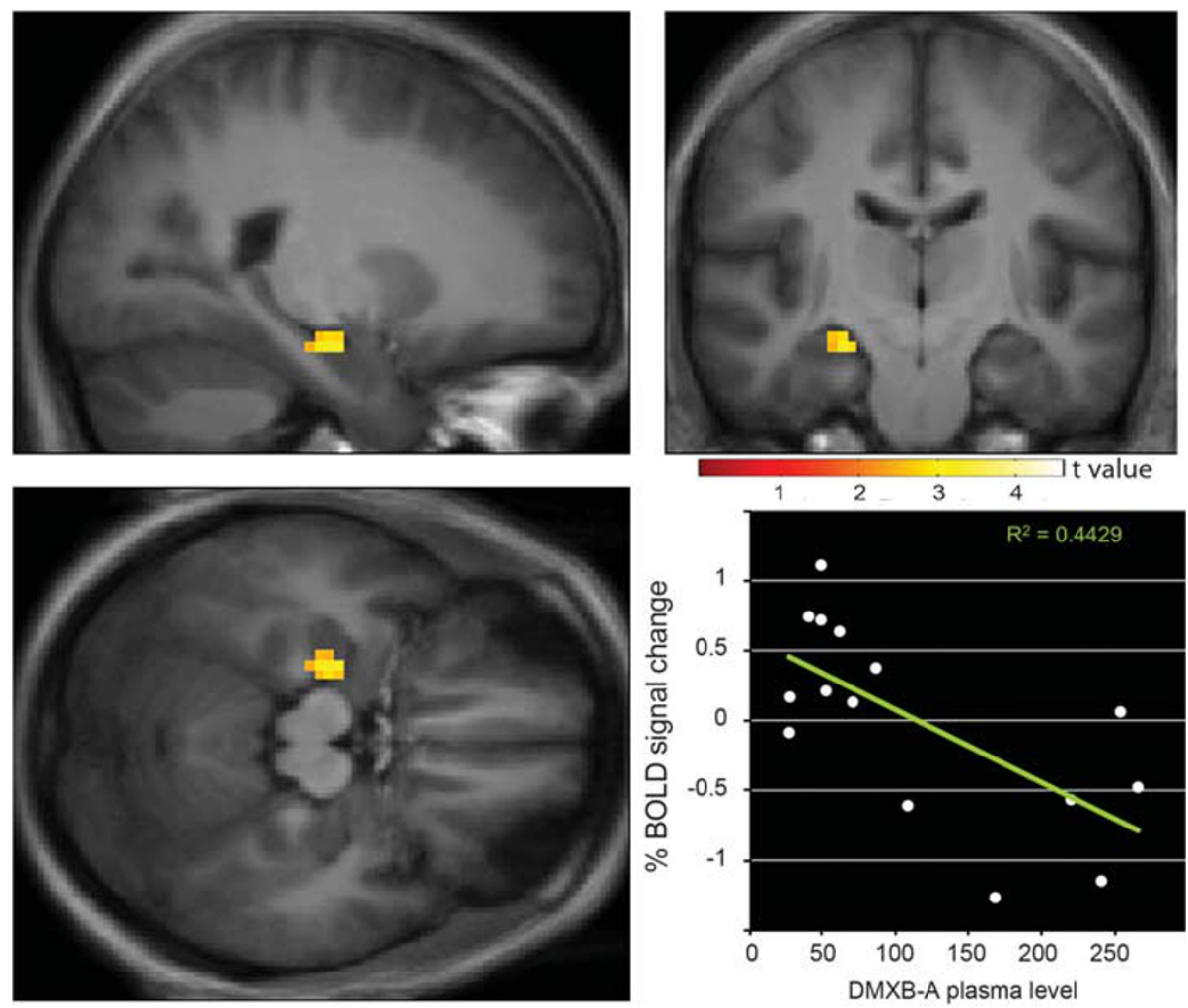

Figure 3 Inverse correlation between $150 \mathrm{mg}$ DMXB-A plasma levels and task-related hippocampal activity. Statistical map thresholded at $p<0.0 \mathrm{I}$ for visualization and overlaid onto the group average anatomical image. Data are shown in the radiological convention (R on $L$ ).

inverted-U shaped dose-response curve in the phase 1 study (Olincy et al, 2006). Phase 2 neurocognitive results were equivocal because of practice effects on the neurocognitive battery. The imaging data did not show order effects that indicated that patients' responses were changing because of familiarity with the test situation. The clear difference in effect between doses demonstrated in the fMRI experiments suggests that the lower dose should now be eliminated from further consideration. The inverse correlation - increasing plasma concentration and decreasing hippocampal activity - within the higher dose range also supports moving to higher doses.

Second, effects of nicotinic agonists (including DMXB-A) are susceptible to desensitization when given continuously at relatively high concentrations. In this study, patients received the drug twice daily. As DMXB-A's half-life is $2 \mathrm{~h}$, there was opportunity for patients' receptors to return to the native state prior to the next dose. The brain concentrations of DMXB-A attained in previously reported animal and clinical studies were small relative to the concentrations required during in vitro experiments to elicit simultaneous activation of all $\alpha 7$-nicotinic receptors (Kem et al, 2004). This suggests that at conventional therapeutic doses, DMXB-A and other $\alpha 7$-nicotinic agonists affect cognitive processes by activating only a small fraction of the total number of $\alpha 7$-nicotinic receptors present, at low concentrations where most $\alpha 7$-nicotinic receptors are not occupied by the agonist (Papke and Thinschmidt, 1998; Papke and Porter-Papke, 2002). DMXB-A is only a partial agonist at human $\alpha 7$-nicotinic receptors, a property that also may be protective against tachyphylaxis (Briggs et al, 1995).

Future study with this therapeutic principle, either with DMXB-A or with another nicotinic agonist, will involve giving long-acting drugs or sustained release formulations to achieve longer periods of activation. The risk of tachyphylaxis will increase accordingly. This imaging paradigm will allow us to determine whether or not the intended biological effect is occurring.

\section{ACKNOWLEDGEMENTS}

This study was supported by the VA Biomedical Laboratory and Clinical Science Research and Development Service; the Mental Illness Research, Education, and Clinical Centers of Veterans Integrated Service Networks 5 and 19; NIMH Grants MH-061412 and MH-086383; the National Association for Research in Schizophrenia and Affective Disorders; the Dana Foundation; and by the Institute for Children's Mental Disorders.

\section{DISCLOSURE}

Dr Freedman has a patent through the Department of Veterans Affairs (VA) on the CHRNA7 gene sequence. Drs Kem and Soti have patents through the University of Florida on the manufacture and use of DMXB-A and have a research grant from CoMentis; Drs Olincy and Johnson receive research support from Lundbeck. The other authors have no conflict of interest. 


\section{REFERENCES}

Andreasen NC (1982). Negative symptoms in schizophrenia. Definition and reliability. Arch Gen Psychiatry 39: 784-788.

Breese CR, Adams C, Logel J, Drebing C, Rollins Y, Barnhart M et al (1997). Comparison of the regional expression of nicotinic acetylcholine receptor $\alpha 7$ mRNA and $\left[{ }^{125} \mathrm{I}\right]-\alpha$-bungarotoxin binding in human postmortem brain. J Comp Neurol 387: 385-398.

Briggs CA, McKenna DG, Piattoni-Kaplan M (1995). Human alpha-7 nicotinic acetylcholine receptor responses to novel ligands. Neuropharmacology 34: 583-590.

De Fiebre CM, Meyer EM, Henry JC, Muraskin SI, Kem WR, Papke RL (1995). Characterization of a series of anabaseinederived compounds reveals that the 3-(4)-dimethylaminocinnamylidine derivative is a selective agonist at neuronal nicotinic alpha-7/125 I-alpha-bungarotoxin receptor subtypes. Mol Pharmacol 47: 164-171.

Frazier CJ, Rollins YD, Breese CR, Leonard S, Freedman R, Dunwiddie TV (1998). Acetylcholine activates an alpha-bungarotoxin-sensitive nicotinic current in rat hippocampal interneurons, but not pyramidal cells. J Neurosci 18: 1187-1195.

Freedman R, Coon H, Myles-Worsley M, Orr-Urtreger A, Olincy A, Davis A et al (1997). Linkage of a neurophysiological deficit in schizophrenia to a chromosome 15 locus. Proc Natl Acad Sci USA 94: 587-592.

Freedman R, Olincy A, Buchanan RW, Harris JG, Gold JM, Johnson L et al (2008). Initial phase 2 trial of a nicotinic agonist in schizophrenia. Am J Psychiatry 165: 1040-1047.

Kem WR, Mahnir VM, Prokai L, Papke RL, Cao X, LeFrancois S et al (2004). Hydroxy metabolites of the Alzheimer's drug candidate 3-[(2,4-dimethoxy)benzylidene]-anabaseine dihydrochloride (GTS-21): their molecular properties, interactions with brain nicotinic receptors, and brain penetration. Mol Pharmacol 65: 56-67.

Mahnir V, Lin B, Prokai-Tatrai K, Kem WR (1998). Pharmacokinetics and urinary excretion of DMXB-A (GTS-21), a compound enhancing cognition. Biopharm Drug Dispos 19: 147-151.
Malaspina D, Storer S, Furman V, Esser P, Printz D, Berman A et al (1999). SPECT study of visual fixation in schizophrenia and comparison subjects. Biol Psychiatry 46: 89-93.

Martin LF, Kem WR, Freedman R (2004). Alpha-7 nicotinic receptor agonists: potential new candidates for the treatment of schizophrenia. Psychopharmacology 174: 54-64.

Olincy A, Harris JG, Johnson LL, Pender V, Kongs S, Allensworth $\mathrm{D}$ et al (2006). Proof-of-concept trial of an a7 nicotinic agonist in schizophrenia. Arch Gen Psychiatry 63: 630-638.

Overall JE, Gorham DR (1962). The brief psychiatric rating scale. Psychol Rep 10: 799-812.

Papke RL, Porter-Papke JK (2002). Comparative pharmacology of rat and human $\alpha 7 \mathrm{nAChR}$ conducted with net charge analysis. Br J Pharmacol 137: 49-61.

Papke RL, Thinschmidt JS (1998). The correction of alpha7 nicotinic acetylcholine receptor concentration-response relationships in Xenopus oocytes. Neurosci Lett 256: 163-166.

Simpson GM, Angus JWS (1970). A rating scale for extrapyramidal side effects. Acta Psychiatr Scand 45: 11-19.

Sobotka S, Nowicka A, Ringo JL (1997). Activity linked to externally cued saccades in single units recorded from hippocampal, parahippocampal, and inferotemporal areas of macaques. J Neurophysiol 78: 2156-2163.

Stevens KE, Kem WR, Mahnir VM, Freedman R (1998). Selective $\alpha_{7}$-nicotinic agonists normalize inhibition of auditory response in DBA mice. Psychopharmacology 126: 320-327.

Tanabe J, Tregellas J, Miller D, Ross RG, Freedman R (2002). Brain activation during smooth-pursuit eye movements. Neuroimage 17: 1315-1324.

Tregellas J, Tanabe JL, Martin LF, Freedman R (2005). fMRI of response to nicotine during a smooth pursuit eye movement task in schizophrenia. Am J Psychiatry 162: 391-393.

Tregellas JR, Tanabe JL, Miller DE, Ross RG, Olincy A, Freedman R (2004). Neurobiology of smooth pursuit eye movement deficits in schizophrenia: an fMRI study. Am J Psychiatry 161: 315-321. 